\title{
Gross motor function in children with Congenital Zika Syndrome from Rio de Janeiro, Brazil
}

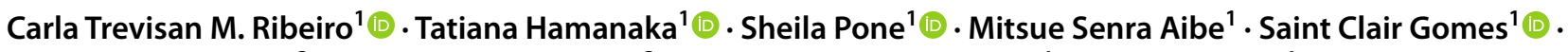

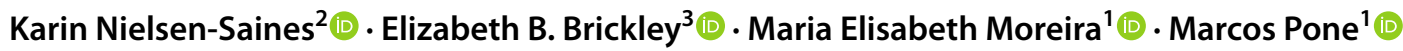

Received: 18 March 2021 / Revised: 26 August 2021 / Accepted: 22 September 2021 / Published online: 1 October 2021

(c) The Author(s) 2021

\begin{abstract}
Congenital Zika Syndrome (CZS) is characterized by many impairments especially in the central nervous system, potentially compromising neurodevelopment and causing significant morbidity in affected children. The aim was to assess gross motor function in children with CZS. This was a cross-sectional investigation nested within a prospective cohort study of children with CZS based in a Brazilian referral hospital in Rio de Janeiro. Between March/2017 and February/2018, we performed gross motor function assessments using the Gross Motor Function Classification (GMFCS) and the Gross Motor Function Measure (GMFM), estimating the mean and standard deviation of GMFM scores among GMFCS groups. The study sample included 72 children, with a median age of 13 months (7-25). Of these, 63 (87.5\%) had severe motor impairment, $3(4 \%)$ had moderate impairment, and $6(8 \%)$ had mild impairment. The mean GMFM score for each group was respectively 11.6, 26.1, and 81.6, with statistically significant differences (p-value $<0.001$ ). Severely affected children only achieved head control in the sitting posture when supported. Children with milder forms were able to develop walking skills.

Conclusion: Most children with CZS have major motor disabilities and a poor prognosis. Better understanding of limitations and functionality in children with CZS can serve as a prognostic guide in their management.
\end{abstract}

\section{What is Known:}

- Severe motor impairment was present in 63 (87.5\%) children with CZS.

- The degree of neurological impairment was inversely associated with motor performance.

What is New:

- Microcephaly was more frequent among children with severe gross motor function impairment.

- Children with CZS have major motor disabilities and a poor prognosis.

Keywords Zika $\cdot$ Zika virus infection $\cdot$ Neurodevelopmental disorders

Fetal infection by Zika virus (ZIKV) may disrupt brain embryogenesis and result in microcephaly and other neurological abnormalities, including subcortical parenchymal

Communicated by Nicole Ritz

Carla Trevisan M. Ribeiro

carla.ribeiro@iff.fiocruz.br

1 National Institute of Women, Children and Adolescents Health Fernandes Figueira, Oswaldo Cruz Foundation (IFF-Fiocruz), Avenida Rui Barbosa, 716, Flamengo, Rio de Janeiro 22250-020, Brazil

2 David Geffen School of Medicine, University of California, Los Angeles, USA

3 School of Hygiene \& Tropical Medicine, London, UK calcifications, ventriculomegaly, and cortical migration abnormalities [1, 2]. Congenital Zika Syndrome (CZS) is characterized by the presence of these central nervous system (CNS) abnormalities as well as osteoarticular deformities and hearing and/or visual impairments, which may compromise neurodevelopment and cause significant morbidity in affected children [1-5]. These sensory and motor alterations are similar to those present in children with cerebral palsy (CP) who have musculoskeletal and neural restrictions that impede the acquisition of functional abilities [6-8]. Motor changes in CZS are often associated with cognitive, communication, and behavioral disorders that prevent children from reaching developmental milestones $[6,7]$. The Gross Motor Function Measure (GMFM) was designed and validated to assess changes in gross motor function over time 
in children with CP $[9,10]$ and it can be an efficient tool for assessing neurodevelopment of children with CZS. The Gross Motor Function Classification System (GMFCS) is used to define a child's ability and functional limitations, serving as a guide to inform motor prognosis [11]. The purpose of this study was to assess gross motor function in children with CZS, a recently described clinical condition with scarce data available on the neurodevelopmental trajectories of affected populations.

\section{Methods}

The study was conducted at the National Institute of Women, Children and Adolescents Health Fernandes Figueira, Oswaldo Cruz Foundation (IFF-Fiocruz), a major referral center for congenital infections in Rio de Janeiro, Brazil. Infants with presumed CZS were enrolled in a longitudinal cohort during the ZIKV outbreak in Brazil in 2015-2016. The study population was comprised of children with CZS who were evaluated in this study once between the ages of 7 and 25 months from March 2017 to February 2018. This cross-sectional study nested within a cohort was approved by the IFF-Fiocruz institutional review board (Plataforma Brasil, CAAE: 52,675,616.0.0000.5269). Parents or guardians provided written informed consent for their children's participation.

The study sample included children, older than 6 months, born during or immediately after the ZIKV outbreak in Rio de Janeiro, Brazil. Children with presumed/probable CZS were defined as having characteristic clinical features, neurological manifestations, neuroimaging abnormalities, and/ or ocular alterations typical of congenital ZIKV infection, assessed by neurofunctional physical therapy. Children with confirmed CZS had all the features of children with presumed/probable CZS plus laboratory confirmation of maternal or fetal infection. The characteristic clinical phenotype of CZS included: microcephaly, overlapping cranial sutures, partially collapsed skull, prominent occipital bone, redundant scalp skin, and/or severe neurological impairment, with or without accompanying arthrogryposis. Neurological manifestations included: irritability, hypertonia and spasticity, hypotonia, hyperreflexia, and/or seizures. The alterations considered in the neuroimaging exams included: intracranial calcifications, ventriculomegaly and extra-axial fluid, abnormal gyral pattern (e.g., polymicrogyria), decreased cerebral parenchymal volume, cortical atrophy and malformations, cerebellar or cerebellar vermis hypoplasia, delayed myelination, and hypoplasia of the corpus callosum. The typical ocular alterations included: hypoplasia or pallor of the optic nerve, increased cup-to-disc ratio, chorioretinal atrophy or scar, pigment mottling, hemorrhagic retinopathy and abnormal retinal vasculature.
Children with arthrogryposis or other osteoarticular congenital malformations were excluded from the analysis to reduce confounding factors in motor assessment. Other congenital infections within the TORCH group, such as toxoplasmosis, rubella, cytomegalovirus infection, and herpes simplex virus infection were excluded by negative serology. Microcephaly was defined as head-circumference at birth below -2 standard deviations for gestational age and gender (z-score -2). Similarly, severe microcephaly was defined as a head-circumference at birth below $\mathrm{z}$-score -3 . We used the INTERGROWTH-21st Project online software to calculate $\mathrm{Z}$-scores for birth measurements based on gestational age and sex (http://intergrowth21.ndog.ox.ac.uk) [12-14].

Each child was evaluated once by a GMFM-trained physical therapist (ICC $>0.98$ for trained) and was classified according to the GMFCS- 88 . We assessed 88 items in the following dimensions: (A) Lying and rolling; (B) Sitting; (C) Crawling and kneeling; (D) Standing; and (E) Walking, running, and jumping. Children were also classified in 5 progressive levels of independence and functionality by GMFCS total score. Level I indicates the ability to walk without any restrictions. Level II indicates some limitations in gait. At level III, children need some assistance to walk. At level IV, children need assistive technology equipment to move. Level $\mathrm{V}$ is reserved for children with severe movement limitations even with the aid of modern technology and total dependence for performance of routine tasks. For analysis purposes, we classified children at levels I and II as having mild impairment, at level III as having moderate impairment, and at levels IV and V as severe impairment; and the GMFM were described quantitatively according to the standardization of the scale with percentage of each dimension and total percentage. Data were analyzed as the mean and standard deviation of these percentages according to the groups of functional impairment [15-17]. Additional information on age, sex, head circumference at birth and prenatal ZIKV infection confirmation (i.e., maternal infection during pregnancy or congenital infection confirmed at birth) was extracted from medical records.

Data was stored in an EPIINFO 7 database. The data analysis was descriptive with measures of central tendency and position and measures of dispersion. GMFM results were analyzed by the Gross Motor Ability Estimator software (GMAE). Analysis of gross motor function occurred through the mean and standard deviation by disease severity groups. Kruskal-Wallis tests were used to test for differences between groups for continuous variables and chi-square tests were used for categorical variables. The Spearman correlation index was used to verify the association between the GMFCS functional classification categories and the GMFM88 total scores (percentage). Statistical Package for Social Sciences (SPSS) version 20.0 was used, and the significance level of $\alpha=0.05$ was considered. 
Table 1 Description of study population according to GMFCS classification as mild, moderate, and severe impairment groups

\begin{tabular}{llllll}
\hline & & GMFCS & & Total \\
\cline { 3 - 4 } & & Mild & Moderate & Severe & \\
\hline Number of participants & & $\mathrm{n}=6$ & $\mathrm{n}=3$ & $\mathrm{n}=63$ & $\mathrm{~N}=72$ \\
Mean (SD) age in months & & $14.5(1.4)$ & $11.7(0.6)$ & $14.1(4.7)$ & $13.9(4.4)$ \\
Sex & Female & $3(50.0 \%)$ & $1(33.3 \%)$ & $29(46.0 \%)$ & $33(45.8 \%)$ \\
& Male & $3(50.0 \%)$ & $2(66.7 \%)$ & $34(54.0 \%)$ & $39(54.2 \%)$ \\
Microcephaly & Yes & $2(33.3 \%)$ & 0 & $51(81.0 \%)$ & $53(73.6 \%)$ \\
Microcephaly severity & No & $4(66.7 \%)$ & $3(100 \%)$ & $12(19.0 \%)$ & $19(26.4 \%)$ \\
& Moderate & $1(50.0 \%)$ & 0 & $16(31.4 \%)$ & $17(32.1 \%)$ \\
ZIKV detection & Severe & $1(50.0 \%)$ & 0 & $35(68.6 \%)$ & $36(67.9 \%)$ \\
(RT-PCR) & Yes & $4(66.7 \%)$ & $2(66.7 \%)$ & $25(39.7 \%)$ & $31(43 \%)$ \\
Physical therapy & No & $2(33.3 \%)$ & $1(33.3 \%)$ & $38(60.3 \%)$ & $41(57 \%)$ \\
GMFCS levels & Yes & $4(66.7 \%)$ & $3(100 \%)$ & $56(88.9 \%)$ & $65(87.5 \%)$ \\
Mean (SD) GMFM & No & $2(33.3 \%)$ & 0 & $7(11.1 \%)$ & $9(12.5 \%)$ \\
\hline
\end{tabular}

GMFCS, gross motor function classification system; RT-PCR, reverse transcription-polymerase chain reaction; ZIKV, Zika virus

\section{Results}

Seventy-two children with CZS were evaluated, of whom 39 (54\%) were male, with ages ranging from 7 to 24 months (median age 13 months). Prenatal ZIKV infection was confirmed via real-time reverse-transcriptase-polymerase-chain reaction (RT-PCR) assays in $31(43 \%)$ cases for either the mother, neonate, or both.

Most children, 63 (88\%) cases, had severe gross motor impairment, 3 (4\%) had moderate impairment, and 6 (8\%) had mild impairment. There was no significant difference between groups regarding age and sex (Table 1). Microcephaly was more frequent in the severe gross motor function impairment group, occurring in 51 (83\%) cases, with a statistically significant difference between groups (p-value $=0.001$, Chi-squared test). In fact, $35(56 \%)$ children from this group presented with severe microcephaly. Nevertheless, microcephaly was also observed in 2 children with mild gross motor function impairment. Most children underwent physical therapy and started treatment by a mean age of 4.7 months (range: 1 to 16 months; SD: 3.0).

The mean GMFM score was 81.6 (ranging from 75 to 90 ) in the mild impairment group, 26.1 (19 to 33) in the moderate impairment group and 11.6 (4 to 22 ) in the severe impairment group. There was a statistically significant difference between groups ( $p$-value $<0.001$, ANOVA test). The mean total GMFM score was 17.6 and the median (range) score was 11.5 (3.7-90.3). Only 9 (12.5\%) children did not undergo physical therapy. Spearman correlation index revealed a negative and statistically significant association between GMFCS groups and GMFM score $(-0.6$; p-value $<0.001)$.

Regarding GMFM results, the severely impaired group, which corresponded to $88 \%$ of the children, was functionally limited and therefore only scored in the first two dimensions for (A) lying and rolling (mean score $=38.8$ ), and (B) sitting (mean score $=16.8$ ). In comparison, children in the moderately impaired group had higher scores in the second dimension (sitting), with many being able to remain seated without upper limb support, and some even had some abilities on dimension $\mathrm{C}$ (crawling and kneeling), with a mean score of 4.0 in this dimension. On the other hand, children in the mildly impaired group had fewer limitations in their gross motor skills, and a subset were able to reach some abilities of dimension $\mathrm{E}$ (walking, running and jumping), with a mean score of 36.4 (Table 2).

\section{Discussion}

The study results demonstrate an association between the degree of neurological impairment, as indicated by GMFCS level, and gross motor performance in children with CZS, as previously described in the CP literature [8, 12, 18-20]. As described in CP studies [15, 21], children with severe impairment (GMFCS levels IV and V) also had severe impairment of gross motor function, with a low mean GMFM score. Severely impaired children in our study were unable to achieve more advanced postures (such as sitting, 
Table 2 Mean (SD) GMFM scores in each dimension according to gross motor function impairment groups

\begin{tabular}{llll}
\hline & $\begin{array}{l}\text { Mild } \\
\mathrm{n}=6\end{array}$ & $\begin{array}{l}\text { Moderate } \\
\mathrm{n}=3\end{array}$ & $\begin{array}{l}\text { Severe } \\
\mathrm{n}=63\end{array}$ \\
\hline $\begin{array}{l}\text { Dimension A } \\
\text { (lying and rolling) }\end{array}$ & $100(0)$ & $84.3(16.7)$ & $38.3(14.9)$ \\
$\begin{array}{l}\text { Dimension B } \\
\text { (sitting) }\end{array}$ & $98.6(2.2)$ & $42.2(12.5)$ & $16.8(6.2)$ \\
$\begin{array}{l}\text { Dimension C } \\
\text { (crawling and kneeling) }\end{array}$ & $90.0(11.3)$ & $4.0(6.9)$ & $0(0)$ \\
$\begin{array}{l}\text { Dimension D } \\
\text { (standing) }\end{array}$ & $83.3(9.3)$ & $0(0)$ & $0(0)$ \\
$\begin{array}{l}\text { Dimension E } \\
\text { (walking, running, and jump- } \\
\text { ing) }\end{array}$ & $36.4(15.7)$ & $0(0)$ & $0(0)$ \\
\hline
\end{tabular}

Means and standard deviation in parentheses

crawling and walking), with some only reaching head control in the sitting posture when supported by the therapist. Also of note, despite neuromotor impairments, some children with milder forms of CZS, especially regarding muscle tone, were able to reach gait and other more elaborate functions in orthostatic posture, such as leaping and kicking a ball.

Using the GMFCS tool, $88 \%$ of children were classified in levels IV or $\mathrm{V}$, indicating a more severe motor impairment and the need for assistance for performance of daily tasks. Such impairment was similar to that described in other case series of CZS [22-24]. In a study with 77 children with CZS, [22] observed that $97.4 \%(n=75)$ had severe functional impairment (GMFCS IV and V) and only $2.6 \%(\mathrm{n}=2)$ had mild functional impairment (GMFCS I); however, the inclusion of children with arthrogryposis may have increased the frequency of serious abnormalities. Carvalho et al. [23] evaluated 69 children with CZS, of whom 4 had arthrogryposis, and observed that only $7 \%(\mathrm{n}=5)$ were classified as GMFCS I to III and 93\% $(n=64)$ were classified as GMFCS IV or V. A recent study [24] with 110 children born with CZS had similar findings, where $90.2 \%$ of children had GMFCS V, $4.9 \%$ had GMFCS IV, $2 \%$ GMFCS III and 2.9\% GMFCS I. The present study did not include children with arthrogryposis in order to avoid interference in GMFM scoring. The literature has already described the association between congenital orthopedic abnormalities and worse neurological outcome [25-27].

Children with CZS and muscle tone, sensory (visual and/ or auditory) or orthopedic (hip dislocation and/or arthrogryposis) abnormalities have greater limitations in performance of daily tasks and are restricted in their ability to participate in age-appropriate activities. This can be further compounded by limited resources restricting access to medication, physical therapy or assistive technology [8]. Ferreira et al. [7] described a variety of structural and functional deficiencies in children with CZS, such as dysphagia, cognitive and language disorders, altered muscle tone, decreased control of voluntary movements and decreased joint mobility. These deficiencies deeply impacted performance of daily activities, as fine motor development was not age-appropriate in more than $90 \%$ of the children, who were also unable to walk. Another study by [19] analyzed 24 children with CZS and identified hyperreflexia in $100 \%$ of the sample, increased muscle tone in $95 \%$ and clonus in $77 \%$. No child was able to stand or walk even with support, which denotes a significant motor delay. The present study results have demonstrated similar findings.

In the current study, the mean GMFM score was 11.5 (range: 3.7-90.3). Furthermore, there was an inverse association between GMFCS groups and GMFM scores, indicating the higher the level of GMFCS (greater functional impairment), the lower the GMFM score. Melo et al. [18] analyzed a group of children with CZS between 5 and 29 months of age and showed a mean GMFM score of 6.5 (range: 2-82.9), which is lower than that observed in this study.

GMFM and GMFCS are promising tools for improving the understanding of limitations and prognosis of children with CZS. They serve as a guide for tailoring care and informing health care and educational policies and priorities $[10,12]$. The poor motor prognosis in our study participants raises concerns about prospects for the group's full participation in the daily activities of society without substantial long-term support.

We used GMFM-88 instead of the reduced version of the scale, GMFM-66, since the full version is more sensitive for detection of alterations in dimensions A and B [28]. Younger and/or severely affected children are mostly able to reach developmental milestones in the first two dimensions; the research study sample was evaluated at a young age (mean of 13.9 months) and was mostly considered severely impaired. Therefore, GMFM-88 appears to be an appropriate tool to evaluate gross motor function in children with CZS.

As seen in Table 2, the group with severe impairment according to GMGCS levels, scored only in the first two dimensions (A) lying and rolling and (B) sitting, which demonstrates that they had a worse prognosis, with difficulties in performing simple tasks independently. Children in the group with moderate impairment managed to remain seated without support from the upper limb and even had some skills in dimension $\mathrm{C}$ (crawling and kneeling). These children have the ability to perform postural shifts with greater independence, but they may present postural compensations, like to sit with lumbar hyperkiohosis [23, 29]. Children in the group with mild impairment managed to score in all dimensions, and some of them were already walking. These results are expected, especially for those in the severe group, and have been observed in other studies of children with CZS $[6,8,22]$.

The presence of microcephaly at birth is another important factor for the impact of ZIKV congenital infection on neurological development [3, 6, 19, 30, 31]. Gordon-Lipkin 
et al. [32] have demonstrated that congenital microcephaly is associated with gross motor development abnormalities in $65 \%$ of cases [31]. Microcephaly in CZS is caused by a disruptive process [7, 13]. Severe microcephaly is caused by brain growth arrest, mainly affecting the frontal, temporal and parietal lobes [7, 32]. There is a close relationship between neuromotor development delay and the degree of microcephaly, as the impaired head growth is indicative of the extent of brain damage $[2,8,33,34]$. The study results corroborate these findings, since severe microcephaly was more frequent in the group of children classified as severely impaired by GMFCS.

It is important that longitudinal cohort studies evaluating sensorineural development use standardized scales. A recent study in the northeastern region of Brazil was the first to analyze motor development of children with CZS over time; this study reported advances in motor development in the first 18 months of age [22]. However, at 24 months of age, most children had reached $90 \%$ of their final motor abilities [22].

Although the present study was cross-sectional, prospective studies of children with CZS are essential for the understanding of the natural history of congenital ZIKV infection, motor development of affected children, and their functional impairments. Although exclusion of children with arthrogryposis can be considered a study limitation in the present study, their inclusion would have negatively impacted gross motor development results in CZS. Another limitation the asymmetrical distribution of participants between groups, which may have affected our statistical analysis.

\section{Conclusion}

Low mean GMFM scores in this population, where most children were classified as GMFCS IV and V, demonstrate the severe functional impairment and poor prognosis of children born with CZS. Our results highlight the pressing need for attention to the neurodevelopment of this population with the implementation of early stimulation programs, public health policies and inclusive education, in order to reduce the burden of disease and allow for better motor and sensory development in affected children.

\section{Declarations}

Conflict of interest The authors declare no competing interests.

Open Access This article is licensed under a Creative Commons Attribution 4.0 International License, which permits use, sharing, adaptation, distribution and reproduction in any medium or format, as long as you give appropriate credit to the original author(s) and the source, provide a link to the Creative Commons licence, and indicate if changes were made. The images or other third party material in this article are included in the article's Creative Commons licence, unless indicated otherwise in a credit line to the material. If material is not included in the article's Creative Commons licence and your intended use is not permitted by statutory regulation or exceeds the permitted use, you will need to obtain permission directly from the copyright holder. To view a copy of this licence, visit http://creativecommons.org/licenses/by/4.0/.

\section{References}

1. Wheeler AC, Ventura CV, Ridenour T et al (2018) Skills attained by infants with congenital Zika syndrome: Pilot data from Brazil. PLoS One 13(7):e0201495. Published 2018 Jul 26. https://doi. org/10.1371/journal.pone.0201495

2. Moore CA, Staples JE, Dobyns WB et al (2017) Characterizing the pattern of anomalies in congenital Zika syndrome for pediatric clinicians. JAMA Pediatr 171(3):288-295. https://doi.org/10. 1001/jamapediatrics.2016.3982

3. Calvet G, Aguiar RS, Melo ASO et al (2016) Detection and sequencing of Zika virus from amniotic fluid of fetuses with microcephaly in Brazil: a case study. Lancet Infect Dis 16(6):653660. https://doi.org/10.1016/S1473-3099(16)00095-5

4. World Health Organization (2016) WHO statement on the first meeting of the international health regulations (2005) (IHR 2005). Emergency committee on Zika virus and observed increase in neurological disorders and neonatal malformations

5. Marques FJP, Teixeira MCS, Barra RR et al (2018) Children born with congenital Zika syndrome display atypical gross motor development and a higher risk for cerebral palsy. J Child Neurol 34(2):81-85

6. Saad T, PennaeCosta AA, de Góes FV et al (2018) Neurological manifestations of congenital Zika virus infection. Childs Nerv Syst 34(1):73-78. https://doi.org/10.1007/s00381-017-3634-4

7. Ferreira HNC, Schiariti V, Regalado ICR et al (2018) Functioning and disability profile of children with microcephaly associated with congenital Zika virus infection. Int J Environ Res Public Health 15(6):1107. Published 2018 May 29. https://doi.org/10. 3390/ijerph15061107

8. Rosenbaum P, Paneth N, Leviton A et al (2007) A report: the definition and classification of cerebral palsy April 2006 [published correction appears in Dev Med Child Neurol. 2007 Jun; 49(6):480]. Dev Med Child Neurol Suppl 109:8-14

9. Palisano RJ, Avery L, Gorter JW, Galuppi B, McCoy SW (2018) Stability of the gross motor function classification system, manual ability classification system, and communication function classification system. Dev Med Child Neurol 60(10):1026-1032. https:// doi.org/10.1111/dmcn.13903

10. Russell D (2002) Rosenbaum Pl, Avery Lm. Mac Keith Press, Lane M. Gross Motor Function Measure. User's manual. London

11. Rosenbaum P, Walter SD, Hanna SE et al (2002) Prognosis for gross motor function in cerebral palsy: creation of motor development curves. JAMA 288(11):1357-1363. https://doi.org/10.1001/ jama.288.11.1357

12. Villar J, Altman DG, Purwar M et al (2013) International fetal and newborn growth consortium for the 21st century. The objectives, design and implementation of the INTERGROWTH-21st Project. BJOG 120(suppl 2):9-26. https://doi.org/10.1111/1471-0528.12047

13. Dain Gandelman Horovitz D, da Silva Pone MV, Moura Pone S, Dias Saad Salles TR, Bastos Boechat MC (2016) Cranial bone collapse in microcephalic infants prenatally exposed to Zika virus infection. Neurology 87(1):118-119. https://doi.org/10.1212/ WNL.0000000000002814

14. Van der Linden V, Pessoa A, Dobyns W et al (2016) Description of 13 infants born during October 2015-January 2016 with congenital Zika virus infection without microcephaly at birth - Brazil. MMWR Morb Mortal Wkly Rep 65(47):1343-1348. Published 2016 Dec 2. https://doi.org/10.15585/mmwr.mm6547e2 
15. Chagas PSC, Defilipo EC, Lemos RA, Mancini MC, Frônio JS, Carvalho RM (2008) Classificação da função motora e do desempenho funcional de crianças com paralisia cerebral. Rev Bras Fisioter 12(5):409-416

16. Mancini MC, Alves ACM, Schaper C et al (2004) Gravidade da paralisia cerebral e desempenho funcional. Rev Bras Fisioter 8(3):253-260

17. Wood E, Rosenbaum P (2000) The gross motor function classification system for cerebral palsy: a study of reliability and stability over time. Dev Med Child Neurol 42(5):292-296. https://doi.org/ 10.1017/s0012162200000529

18. Melo A, Gama GL, Da Silva Júnior RA et al (2020) Motor function in children with congenital Zika syndrome. Dev Med Child Neurol 62(2):221-226. https://doi.org/10.1111/dmcn.14227

19. Alves LV, Paredes CE, Silva GC, Mello JG, Alves JG (2018) Neurodevelopment of 24 children born in Brazil with congenital Zika syndrome in 2015: a case series study. BMJ Open 8(7):e021304. Published 2018 Jul 16. https://doi.org/10.1136/bmjopen-2017-021304

20. Miranda-Filho Dde B, Martelli CM, Ximenes RA et al (2016) Initial description of the presumed congenital Zika syndrome. Am J Public Health 106(4):598-600. https://doi.org/10.2105/AJPH.2016.303115

21. Zonta MB (2011) Avaliação funcional na paralisia cerebral. Acta Pediatr Port 42(1):27-32

22. Ventura PA, Lage MLC, de Carvalho AL, Fernandes AS, Taguchi TB, Nascimento-Carvalho CM (2020) Early gross motor development among brazilian children with microcephaly born right after Zika virus infection outbreak. J Dev Behav Pediatr 41(2):134-140. https://doi. org/10.1097/DBP.0000000000000722

23. Carvalho A, Brites C, Mochida G et al (2019) Clinical and neurodevelopmental features in children with cerebral palsy and probable congenital Zika. Brain Dev 41(7):587-594. https://doi.org/10.1016/j. braindev.2019.03.005

24. Cavalcante TB, Ribeiro MRC, Sousa PS, Costa EPF, Alves MTSB, Simões VMF et al (2021) Congenital Zika syndrome: Growtn, clinical and motor development outcomes up to 36 months of age and diferences according to microcephaly at birth. Int J Infect Dis 105:99-408. https://doi.org/10.1016/j.ijid.2021.02.072

25. Moore CA, Staples JE, Dobyns WB, Pessoa A, Ventura CV, Fonseca EB, Ribeiro EM, Ventura LO, Neto NN, Arena JF, Rasmussen SA (2016) Characterizing the pattern of anomalies in congenital Zika syndrome for pediatric clinicians. JAMA Pediatr 171(3):288-295. https://doi.org/10.1001/jamapediatrics.2016.3982
26. Hägglund G, Lauge-Pedersen H, Wagner P (2007) Characteristics of children with hip displacement in cerebral palsy. BMC Musculoskelet Disord 8:101. https://doi.org/10.1186/1471-2474-8-101

27. Carvalho A, Ventura P, Taguchi T, Brandi I, Brites C, Lucena R (2020) Cerebral palsy in children with congenital Zika syndrome: a 2-year neurodevelopmental follow-up. J Child Neurol 35(3):202-207

28. Hielkema T, Hamer EG, Ebbers-Dekkers I et al (2013) GMFM in infancy: age-specific limitations and adaptations. Pediatr Phys Ther 25(2):168-177. https://doi.org/10.1097/PEP.0b013e318288d370

29. Van Eck M, Dallmeijer AJ, Voorman JM, Becher JG (2009) Longitudinal study of motor performance and its relation to motor capacity in children with cerebral palsy. Dev Med Child Neurol 51(4):303-310. https://doi.org/10.1111/j.1469-8749.2008.03263.x

30. Lopes Moreira ME, Nielsen-Saines K, Brasil P et al (2018) Neurodevelopment in Infants Exposed to Zika Virus In Utero. N Engl J Med 379(24):2377-2379. https://doi.org/10.1056/NEJMc1800098

31. Cicuto Ferreira Rocha NA, de Campos AC, Cicuto Ferreira Rocha F, Pereira Dos Santos Silva F (2017) Microcephaly and Zika virus: Neuroradiological aspects, clinical findings and a proposed framework for early evaluation of child development. Infant Behav Dev 49:70-82. https://doi.org/10.1016/j.infbeh.2017.07.002

32. Gordon-Lipkin E, Gentner MB, German R, Leppert ML (2017) Neurodevelopmental outcomes in 22 children with microcephaly of different etiologies. J Child Neurol 32(9):804-809. https://doi. org/10.1177/0883073817707301

33. Schuler-Faccini L, Ribeiro EM, Feitosa IM et al (2016) Possible Association Between Zika Virus Infection and Microcephaly - Brazil, 2015. MMWR Morb Mortal Wkly Rep 65(3):59-62. https://doi.org/ 10.15585/mmwr.mm6503e2

34. Satterfield-Nash A, Kotzky K, Allen J et al (2017) Health and development at age 19-24 months of 19 children who were born with microcephaly and laboratory evidence of congenital Zika virus infection during the 2015 Zika virus outbreak - Brazil, 2017. MMWR Morb Mortal Wkly Rep 66(49):1347-1351. https://doi. org/10.15585/mmwr.mm6649a2

Publisher's Note Springer Nature remains neutral with regard to jurisdictional claims in published maps and institutional affiliations. 